OCEANS'15 MTS/IEEE Washington Proceedings. Systems and Observatories 1, 2015

\title{
Strategy for sustainability of the Joint European Research Infrastructure Network for Coastal Observatories - JERICO
}

\author{
Puillat Ingrid ${ }^{1,}{ }^{*}$, Farcy Patrick ${ }^{1}$, Durand Dominique ${ }^{2}$, Petihakis George ${ }^{3}$, Morin Pascal ${ }^{4}$, \\ Kriegger Magali ${ }^{4}$, Petersen Wilhelm ${ }^{5}$, Tintoré Joaquin ${ }^{6}$, Sorensen Kai ${ }^{7}$, Sparnocchia Stefania ${ }^{8}$, \\ Wehde Henning ${ }^{9}$
}

${ }^{1}$ IFREMER Centre de Brest Plouzané, Brest, France

${ }^{2}$ NIVA, Durand Research \& Consulting, Bergen, Norway

${ }^{3}$ Hellenic Centre for Marine Research - HCMR Heraklion Crete, Greece

${ }^{4}$ CNRS- Station Biologique de Roscoff France

${ }^{5}$ Helmholtz Zentrum Geesthacht - HZG Geesthacht, Germa

${ }^{6}$ CSIC-UIB, SOCIB, Palma, Balearic Island, Spain

${ }^{7}$ Norsk Institutt for Vannforskning- NIVA Oslo, Norway

${ }^{8}$ CNR-ISMAR Trieste, Italy

${ }^{9}$ Institute of Marine Research -IMR Bergen, Norway

* Corresponding author : Ingrid Puillat, email address : Ingrid.puillat@ifremer.fr

\begin{abstract}
:
The JERICO European research infrastructure $(\mathrm{RI})$ is integrating several platform types i.e. fixed buoys, piles, moorings, drifters, Ferryboxes, gliders, HF radars, coastal cable observatories and the associated technologies dedicated to the observation and monitoring of the European coastal seas. The infrastructure is to serve both the implementation of European marine policies and the elucidation of key scientific questions through dedicated observation and monitoring plans. It includes observations of the physical, chemical and biological compartments and aims at a better integration of marine biology with physical and chemical oceanology, through specific interactions with other relevant ocean observing systems that provide complementary observations. The first phase of the implementation of JERICO encompasses setting up, coordination and harmonization, and were performed between 2011 and 2015 in the framework of FP7-JERICO (www.jerico-fp7.eu), a 4-year long infrastructure project co-funded by the European Commission, with 27 partners from 17 European countries under the coordination of IFREMER. The next 4-year phase is to be carried out through the H2020-JERICO-NEXT European project, starting in 2015 and involving 33 scientific and industrial partners. The main objective of the JERICO consortium is to establish a common approach for a pan-European coastal marine observatory network. This is a dynamic and long-lasting effort necessitating continuous work towards harmonization (i.e. design, operation, and maintenance), evolution and extension of the current systems as well as the delivery of data and products to the users. Success relies on a good coordination and follow-up between FP7-JERICO and JERICO-NEXT, and onwards, at both hardware and software levels. More specifically, the existing network and its possible evolution are continuously assessed taking in account the evolution of the user needs, the harmonization effort to be driven, the existing sensors and
\end{abstract}


technologies, their upgrades for integration on dedicated platforms, also the accompanying of under development sensors and/or systems with involvement of providers and stakeholders when possible. Nevertheless, a major issue relates to the sustainability of the infrastructure, both at economical and governance levels, and the capability in integrating the latest technology while preserving the scientific value of the data.

This paper briefly summarizes the work carried out in FP7-JERICO project and drafts strategic aspects of the JERICO-RI sustainability on the long-term. s. We will present the 6 priority scientific areas that are the drivers of JERICO-NEXT scientific strategy and the subsequent technology development to be implemented through dedicated Joint Research Activity Projects. Emphasis is put on how the consortium intends to address long term financial and legal governance structures for the sustainable implementation of JERICO-NEXT infrastructures, as well as access to the infrastructure and associated services and link to stakeholders such as relevant funding agencies and SMEs.

Keywords : Coastal Observatory, Harmonization, valorization, Open access 


\section{INTRODUCTION}

It generally recognized that threats and pressure to coastal areas is increasing; would it refer to increase in coastal population, degradation of coastal habitats, increased pollution, greater demand for non-living resources (renewable energy), or over-fishing that result in declines in ecosystem health and biodiversity. Consequently one experiences an increase demand in data and in-situ observations as the backbone for better understanding the changes in the marine environment and the processes involved. It raised the demand of observation systems suitable to deliver high quality and comprehensive observations, with automated platforms and sensors systems, but not only, as well as autonomy over long periods of time.

Europe is reacting by promoting extensive cooperation for the observation of the global ocean by supporting major infrastructure projects such as EURO-ARGO (www.euroargo.eu) and EMSO (www.emso-eu.org/). One of the key objectives is to create a large international cooperation for observing systems in the global ocean, while the idea of a European Ocean Observing System (EOOS) both in open and coastal ocean has been already formulated [1]. Parts of this integrative approach are the observational systems implemented within the European Global Ocean Observing System (EuroGOOS, http://eurogoos.eu/) for the European waters. Over the last years several European projects have contributed in integrating the in-situ observations towards a system that can serve all the need from the different users.

The exploitation of research-driven marine technologies and data towards innovation, wealth creation and business development is an increasing demand from policy-makers in Europe. This is a crucial driver for decision-making and this is giving an explicit framework, in addition to scientific and environmental concerns, for developing a strategy for coastal observation in Europe.

The rationale for improved observations of coastal seas is to gain a better understanding of variability of biological, chemical and physical processes and to attribute measured changes to causes; natural, anthropogenic or climatic. To strengthen this understanding of the coastal areas processes, to better inform policy makers as well as to contribute to science, we need sustained comprehensive and consistent data on physical, chemical and biological parameters collected at appropriate time and space scales.

Coastal observations are paramount in the marine research because they help understanding marine processes by direct analysis of spatio-temporal data, by assessing the performance of numerical models, and direct assimilation into models. The coastal component of the European marine observing system is to serve both the implementation of European marine policies and the elucidation of key scientific questions through dedicated observation and monitoring plans. It must include observations of the physical, chemical and biological compartments and aims at a better integration of marine biology with physical and chemical oceanology
Despite needs and requirements are relatively similar, from one country to another; significant heterogeneity exists in Europe concerning technological design of observing systems, measured parameters, practices for maintenance and quality control, as well as quality standards for sensors and data exchange. This is because, until a few years ago, the expansion of "coastal observatories" has been driven by domestic interests and mainly undertaken through short-term research projects. Therefore the main challenge for the research community was to harmonize the technologies, increase the coherence and the sustainability of these dispersed infrastructures by sharing know-how through dedicated actions and experiments within a shared pan-European framework. These considerations led 27 leading marine research institutions from 17 European countries (Fig.1) to gather in the FP7-JERICO project (www.jerico-fp7.eu), a 4-year long infrastructure project (2011-2015) co-funded by the European Commission in the $7^{\text {th }}$ Framework Programme. From the $1^{\text {st }}$ Sept. 2015, a new phase begins within the 4-year long H2020JERICO-NEXT European project 9, involving 33 scientific and industrial partners.

The main objective of the JERICO consortium is to establish a common approach for a pan-European coastal marine observatory network. As from a starting point JERICO network mainly integrated 3 kinds of platforms: the fixed ones (buoys, piles, moorings), Ferryboxes and gliders, taking care of their related technologies and data flows.

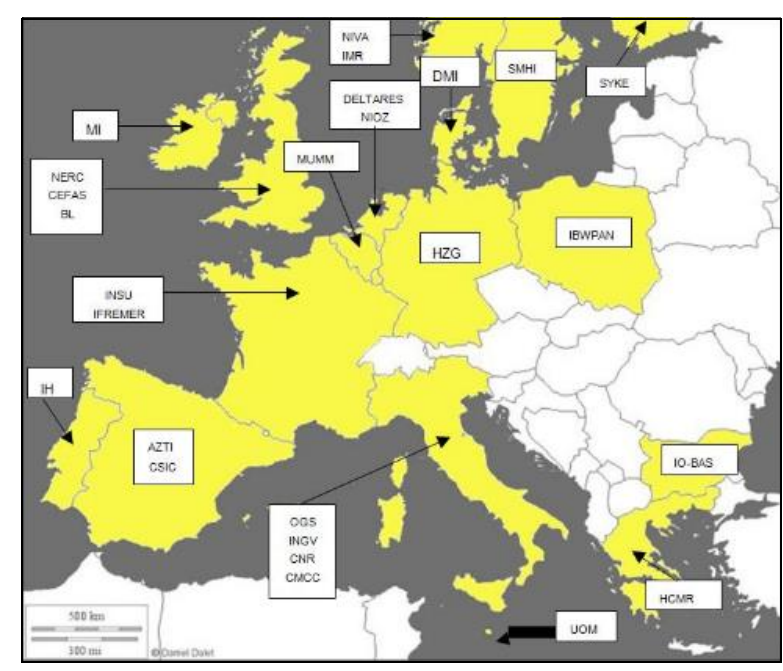

Fig. 1. Map of the institutions involved in FP7-JERICO (2011-2015)

JERICO addresses the challenge of observing the complexity and high variability of coastal areas at PanEuropean level, in the framework established by European Directives (Water Framework Directive: WFD, Marine Strategy Framework Directive: MSFD) and the marine core service of the European Earth observation programme (Copernicus) by:

- $\quad$ setting up an European Research Infrastructure for coastal observations based on existing systems in European coastal and shelf seas, 
- $\quad$ supporting standardization of methodologies for the benefit of data quality, data availability and cost efficiency,

- $\quad$ promoting the cost-effective use of the facilities,

- stimulating the development of new automated systems for the operational monitoring of the coastal marine environment, with focus on the biochemical compartment.

In order to reach this target JERICO is promoting: (i) networking activities addressing standardization issues along the value-chain from sensors to data management, (ii) joint research activities preparing for future development of the RI, (iii) TransNational Access (TNA) to JERICO infrastructures and data.

Networking activities has led to the definitions of best practices for design, implementation, maintenance and data distribution of coastal observing systems, as well as the definition of quality standards. A synthesis of JERICO Best Practices is presented during the IEEE OCEANS'2015 conference [2].

This is a dynamic and long-lasting effort necessitating continuous work towards harmonization (i.e. design, operation, and maintenance), evolution and extension of the current systems as well as the delivery of data and products to the users. Success relies on a good coordination and follow-up between FP7-JERICO and JERICO-NEXT, and onwards, at both hardware and software levels. More specifically, the existing network and its possible evolution are continuously assessed taking in account the evolution of the user needs, the harmonization effort to be driven, the existing sensors and technologies, their upgrades for integration on dedicated platforms, also the accompanying of under development sensors and/or systems with involvement of providers and stakeholders when possible. Nevertheless, a major issue relates to the sustainability of the infrastructure, both at economical and governance levels, and the capability in integrating the latest technology while preserving the scientific value of the data.

In the next section, an overview of the progress achieved during the $1^{\text {st }}$ phase of JERICO will be given, followed by a presentation of the plan for the $2^{\text {nd }}$ phase in section 3 as conclusions and perspectives.

\section{FP7-JERICO: ACHIEVEMENTS AND WAY FORWARD}

Harmonization and strengthening coastal observation systems within the European Global Ocean Observing System (EuroGOOS) has being sought. Opportunities of TransNational Access experiments have been carried out in order to promote the potential of JERICO infrastructures used in synergy. Joint research has been conducted to identify new and strategic technologies to be implemented in the next generation European coastal observatories.

In the following sections details are given on the main progress in terms of geographical integration of systems (section A), integration and harmonization of hardware and software components of the systems (section B) and use of the infrastructures (section $\mathrm{C}$ ).

\section{A. Regional intregration}

In order to address consistency of environmental conditions and scientific challenges, a regional integration has been adopted for JERICO. It has been supported by the use of the pre-existing Regional Ocean Observing Systems (ROOS ${ }^{1}$ ) structure, but has required, involving new partners, and extending the terms of references of the ROOSes to meet JERICO requirements. Important tasks were (1) to establish the state of the art of the existing infrastructure network (hardware) at ROOSes level, (2) to assess its gaps in term of platform locations, measured parameters, sampling frequency etc., then (3) to conclude with recommendations for consolidating and densifying the observation network. This has been performed region per region and synthesized at European level in reports D2.2 and D1.11 (http://www.jerico-fp7.eu/deliverables).

At the European scale, the main bottlenecks which have been identified are the following.

- The sustainability of the existing coastal observational system is one of the main challenges facing the partners within all ROOS region. It should be promoted through a better collaboration framework between actors of core and downstream services, aiming to reach a general coherent system in which the levels of founding (Regional, National and European) are clarified and complementary.

- The implementation of glider repeated sections in all the ROOS regions. Glider activity is mainly developed through research programs with a limited spatial and temporal coverage. It will be necessary to promote a better coordination at the European level to select repeated sections in key zones of the different ROOS regions.

- The durability of the Ferrybox lines or of the ferries affected to repeated lines. During the duration of the project, some lines have been stopped due to change of ferries operating the selected lines or lines which have been stopped by the ferry company. The durability of observations using vessels of opportunity is therefore highly dependent of the maritime companies and can't be easily planned on the long term necessary for observing systems.

Some common recommendations for all regions and priorities for future development have been identified as such hereafter.

- To densify spatial coverage by Ferrybox lines in the Northern seas and to establish Ferrybox network in insufficiently observed areas such as Black Sea and Eastern Mediterranean Sea.

- To systematically add measurement of dissolved oxygen to the existing Ferrybox lines that are not yet equipped with this sensor.

\footnotetext{
1 The Regional Operational Oceanographic Systems (ROOSs) are regional bodies that have been developed out of EuroGOOS Task Teams. They are an essential part of the Organization for the purposes of co-ordination and development of operational collaboration and joint service production in the regions. There are 5 as listed on: http://eurogoos.eu/regional-operationaloceanographic-systems/
} 
- To harmonize the Ferrybox set of sensors by adding recently validated parameters such as carbon system sensors (pCO2, alkalinity) and in the future additional sensors under development such as nutrients, contaminants (bio-sensors, passive sensors, ...) and biological parameters (cyanobacteria, flow cytometer, Fast-repetition rate fluorimetry, ...)

- To implement in all ROOS regions repeated glider sections with a regular frequency to be determined (monthly, seasonally, yearly) and preferentially along existing Ferrybox lines to complement with data obtained along the water column the surface high -frequency data.

- To develop observations of biogeochemical and biological data on fixed platforms which are mainly tide gauges or platforms equipped with physical sensors (temperature, salinity) .A significant effort must be made to implement existing platforms with mature sensors such as dissolved oxygen, fluorometers and turbiditimeters. It is recommended to integrate in the future recently validated sensors such as carbon system sensors (pCO2, alkalinity and $\mathrm{pH}$ ) nutrients, contaminants and biological sensors (flow cytometer, new biological sensors developed in WP10).

- Specific priorities have also been given for each ROOS region.

\section{B. Systems integration: Harmonization from the platforms to the data}

One of the key objectives in JERICO Networking Activities was the harmonization of operation and maintenance methods, acknowledging the fact that European coastal observatories have been developed in a rather uncoordinated way. The national efforts guided by each country's priorities, have led to observatories with very diverse design and architecture. This resulted in the establishment of very different practices for operation and maintenance as expressed in the next two sections. Considering the diversity of practices, from one system to another, and even for a single system in some cases, it became important to propose a federating approach that would guarantee a quality of the outcoming data. This approach is described by the JERICO Label in section III.

\section{1) Harmonization in JERICO}

The degree of "harmonization need" depends to a large extent on the observing platform and the previous European efforts. Thus the Glider community, benefiting from the small variability of available products (few types of gliders) and the European funding opportunities (GROOM project) has moved towards harmonization right from the start, describing more and adopting to a lesser degree, common best practices. One step down the scale is the Ferrybox where although the basic concept and architecture behind the installed systems is more or less the same, the existing technology allows certain degrees of freedom and more "custom designs". However the work done during the FP5 FerryBox project proved to be a very good ground on top of which JERICO started building. For these two platforms (gliders and Ferrybox) there are no obvious harmonization gaps in terms of operation and maintenance although, as technology progresses and new systems are integrated onto observing platforms, new needs for harmonization may appear. It is a dynamic activity, which the community must pursue constantly.

Need for harmonization is strongly expressed within the Fixed Platforms community where a huge variability is found. Standard Operation Procedures (SOP's) followed by each partner were documented and a best practice code was developed. This is reported in JERICO best practice report (deliverable D4.4, www.jerico-fp7.eu/deliverables). Hereafter, the harmonization level reached in JERICO is illustrated for Ferryboxes.

\section{2) Harmonization of Ferryboxes}

The Ferrybox is an automated system used for measuring of physical and biogeochemical parameters in surface waters. It is mounted on Ships Of Opportunity Systems (SOOPs), such as ferries or container ships, on their regular routes across the Sea or on shore-based installations. Water is pumped from a subsurface inlet into the measuring circuit of multiple sensors. An important feature is the regular automated cleaning and antifouling procedure of the box. Data are transmitted and made available in real-time (satellite connection) or after each transect in the harbour (mobile phone connection). The regular transects of physical and biogeochemical observations give detailed insights into the processes and can be used for data assimilation into models [3].

The JERICO best practice report (D4.4) gives an introduction to the most important technical and practical issues concerning Ferrybox installations e.g. which commercial Ferrybox system are available, what are the Ferrybox community uses of core sensors, state-of-the-art of more advances system and what to be specially aware of concerning installation, maintenance, calibration and data handling.

For instance installation indications are given for selection of shipping company, ship type, ship route and how to take in account ship regulations. More specifically technical issues such as water inlet, pump, valves and water supply lines, choice of the system, electrical considerations are expressed. For maintenance and calibration both system and sensors are considered with Quality Assurance.

The Ferrybox data management has certain variation between the members of the European Ferrybox community. But it depends also on the type of parameter. Furthermore, two different paths are needed for the data flow from Ferrybox systems; one for fully automated measurements and one for data from analyses of water samples usually carried out in a laboratory on land. The two paths merge during the quality control process. Data from automated measurements and water sampling should be made freely available to the global oceanographic community after quality control. The existing systems developed by the Intergovernmental Oceanographic 
Commission (IOC) and the International Council for the Exploration of the Sea (ICES) should be used when suitable. One example is that the IOC has a global database on carbon dioxide measurements in the sea. The IOC is the mother organization of the Global Oceanographic Observation System (GOOS) of which EuroGOOS, BOOS and NOOS are parts of. Thus standards set by GOOS should govern standards used in Europe. However, partners of JERICO and MyOcean, have noted that the standards are not well developed for all parameters. A very promising initiative is EMODnet (www.emodnet.eu), a pan-European system for handling marine data. Another promising pan-European initiative, focussed on biodiversity data, is Lifewatch. Many countries have national oceanographic data centres, e.g. the United Kingdom and Sweden. If such a centre exists it should be used to store and distribute data. Another option is to select an institute that collects stores and distributes data for a region. These data centres act as nodes in a European network. Each node is responsible for collecting data, quality control and dissemination of data at the national or regional level. Data should be freely distributed using web feature services and similar techniques. In this way corrected data are automatically distributed to other data centres and users when corrections are made. The data distributed by the nodes should be collected at one, or a few, European Ferrybox data centres. This centre(s) will make the data available in a coherent way and will also visualize the data on maps, in graphs etc. It should be noted that both near real time QC1 data and delayed mode QC3 data should be distributed in the same system.

\section{3) The JERICO Label}

Considering the importance of harmonization issues, the JERICO partners introduced the concept of a JERICO Label in order to:

- acknowledge a consensus on guidelines for best practices in the design, the implementation, the maintenance, the data policy and the valorization of operational coastal observatories;

- $\quad$ achieve fair recognition of the quality of the managed observatories for the partners and all new comers that comply with this Label;

help stakeholders becoming aware of the European interest in the development of high quality coastal observatories; and finally,

- foster a wider market for the industry in sensor technology and platforms based on the agreed recommendations.

The recommendations included in the JERICO Label cover a wide range of issues faced by operators and can be used as a road map towards a European Coastal Observing System (Deliverable D1.8). Following the technological advancements, the recommendations need to be updated regularly especially those related to sensor use, maintenance, calibration, biofouling etc:

- Sensing technologies

- Qualification and testing
- Operating Issues

- Deployment-Installation

- Maintenance

- Biofouling

- Metrology-Calibration

More information is given by Petihakis et al., and is presented during this OCEANS15 conference [2].

In the framework of the FP7-JERICO project the JERICO label was limited to technological harmonization issues. It helped to initiate a harmonization target at European scale considering the heterogeneity of the systems. In order to step forward it is necessary to organize the technology harmonization to match with the scientific and societal needs at European scale, and thereby overcome the limitation arising from the fact that an observing network is usually deployed in a local or regional place to answer specific scientific and societal questions that are specific to an area. As a consequence the main priority for the next steps is to upgrade the JERICO label to make it matching with scientific and policy priorities. Moreover, FP7-Jerico focused upon a limited numbers of key physical and chemical parameters for which good progress in terms of integration and harmonization was achieved. Consensus around biological data and subsequent observing approach and technologies is foreseen to be more difficult to be reached. It is going to be one of the main objectives of the JERICO-Next project.

\section{C. $\quad$ Access to the facilities and Support to research activities and policy}

To support researches and developments JERICO offered two kinds of opportunities: access to the Infrastructure, by opening calls for TranNational Access (TNA), and access to services (mostly to data), by the so called Service Access (SA). Both are described hereafter and next steps are presented.

\section{1) TranNational Access (TNA)}

During its lifetime JERICO offered and supported access to a number of European Coastal Observatories and Calibration Facilities for international research and technology development. The primary objective of the TNA activity was to enable scientists and engineers to freely access coastal infrastructures not available in their own countries. Access to these facilities contributes to

- $\quad$ build a long-term collaboration between users and JERICO partners, facilitating staff exchange and scientific cooperation;

- build an European facility for science dedicated to innovation (new sensors, new automated platforms), open to Europe and also to countries of common regional interest (South Mediterranean, Black Sea, Baltic Sea);

- $\quad$ promote the infrastructure by transferring know-how from the partners to users, with a view to future expansion that will include new partners (possibly also from non-European countries). 
Nineteen out of twenty-four proposals submitted to the three calls for proposal were evaluated and selected by an international Selection Panel. Related projects have received support by JERICO. The operators of the targeted facilities have contributed to these projects by providing all the logistical, technological and scientific support as well as specific training, when needed. TNA can be illustrated through 3 examples, highlighting how the JERICO infrastructure supports science, help developing know-how and methods, as for calibration experiment, and how the gathered information answers crucial questions such as contaminant distribution.

- The CIEBIO TNA-project: "Calibration and intercalibration exercise of bio-geochemical sensors" [4]. The experiment addresses the main scope of performing a calibration and inter-calibration exercise of bio-geochemical sensors to be operationally and routinely deployed on off-shore marine observatories making part on a continuous basis of the marine monitoring network of the Mediterranean Sea. In particular, the first objective consists in enhancing the accuracy of the in-situ observations on a long term basis of dissolved oxygen, chlorophyll-a and turbidity in the Ligurian basin collected by a multi-parametric probes installed on the W1M3A offshore observing system, constituted by the "ODAS Italia 1" spar buoy and by a close subsurface mooring. The opportunity to install carefully calibrated probes will improve the knowledge about the biogeochemical processes in the upper thermocline and can support with real-time quality controlled observations the developing biogeochemical forecast models for both the phases of assimilation and calibration/validation.

- ECCECs TNA-project: "Emerging Chemical Contaminants in European Coasts" [5]. The objectives of this project was to provide a first continental scale, consistent assessment of emerging chemical contaminants occurrence and distribution in European coastal waters and to assess the budget of selected chemical contaminants in the marine water column and investigate the mechanisms controlling their vertical distribution.

- $\quad$ MEDACID TNA-project : "Mediterranean Sea ocean acidification time series experiment" [6]. Coastal waters are badly sampled for carbon dioxide and only some $\mathrm{CO} 2$ sensors have been recently deployed along USA coastal waters and North of Europe. In this project, the user group deployed one of its $\mathrm{pH}$ sensors having a $0.001 \mathrm{pH}$ unit reproducibility, on one of the buoy of the HCMR POSEIDON networks, placed in an important region as it is the east coastal Mediterranean seawater. Calibration experiments were also planned at the HCMR calibration facility. The project will add great value to: a) the development of $\mathrm{pH}$ measuring system, b) the monitoring of ocean acidification in the Mediterranean Sea and c) the JERICO project (both from the point of view of Trans National activities and co-operation and the development of novel and advanced measuring systems).

\section{2) Service Access ( $S A)$}

SA embodies the ultimate goal of the coastal observatories, i.e. the provision of useful data. JERICO made a SA demonstration with 18 nodes of the network delivering their data to the JERICO hub during $\sim 2$ years after January 2013.
Taking into account what has been recommended by the Data Management coordination, the objective was to make the data flowing through the 2 main marine data circulation structures maintained at European level. One structure is MyOcean and in the very near future Copernicus TAC-In situ for near real time data dedicated to operational oceanography needs. The other one is SeadataNet delayed mode data structure based on the NODC -National Ocean Data Centres- network. Practically speaking the data are first integrated in MyOcean then they are transmitted to SeaDataNet which acts as a portal for EMODNET (Fig. 2).

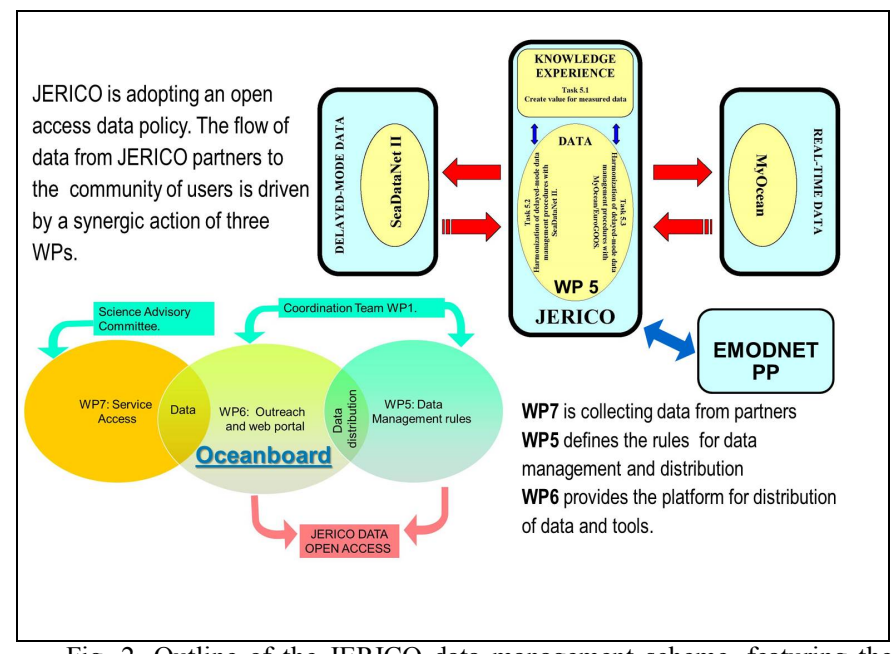

Fig. 2. Outline of the JERICO data management scheme, featuring the driving actions and main information flows.

Data are available on the here above cited data portal as well as on JERICO webpage (on "Data Access" tab, Fig. 3).

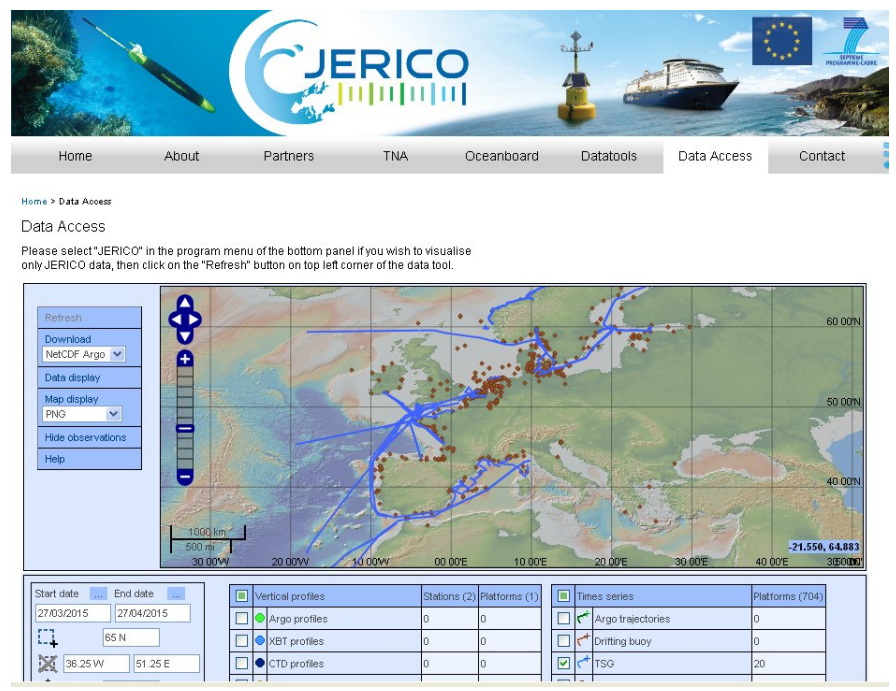

Fig. 3. JERICO web page to access the data

3) Next steps to support coastal Research and developments.

Research and development results carried out during the FP7-JERICO were presented during the dedicated JERICO science Day, in Brest (France) the 28-29 April 2015 (http://jerico-fp7.eu/events/284-jerico-week-and-general- 
assembly-28-30th-april-2015-brest-france). A large part of the results are in a publication process for a special edition in Journal of Marine Systems, for which 13 articles are expected, highlighting the importance of such an infrastructure in developing synergy between several kinds of platforms (mainly 3 in the FP7 project), increasing the quality of the results, integrating knowledge and know-how, and several scientific communities.

These considerations also raised the need to push further ahead the coastal European network to better satisfy scientific and technology expectation, by complementing the existing with other platform types (e.g. HF radars), specific calibration laboratories, but also the observations of additional environmental parameters, addressing specifically the biological compartment. During the lifetime of the project, the contribution of JERICO in fulfilling European policy requirements such as the ones from the Marine Strategy Framework Directive (MSFD) became an important topic. The need for long-term continuous monitoring that can efficiently support both science and policy, was also frequently raised as an issue from technology, sampling and economical point of views. Besides, sustainability is considered to be the most important challenge to be addressed in the future. These conclusions were taken into account to write the JERICONEXT proposal.

\section{CONCLUSIONS AND PERSPECTIVES}

The JERICO community emphasizes that one cannot understand the complexity of the coastal ocean if one do not understand the relationships between the physics, biogeochemistry and biology of coastal systems. Reaching such an understanding requires new technological developments allowing for the continuous monitoring of a larger set of parameters than usually performed. It also requires an a priori definition of the optimal deployment strategy in view of coupling diverse data monitored over very different spatial and temporal scales. This is why JERICONEXT will focus its main effort to the assessment of the interactions between physical, biogeochemical and biological processes, and will work at establishing scientific strategies suitable to address both policy requirements and science.

In JERICO-NEXT the focus is set on the observation of the biological compartment, the valorization of the JERICO products through joint research activities, the maturation and testing of novel and promising technologies, the relationship with stakeholders and relevant SMEs and the sustainability of the infrastructure and of the technological network. The observation of the biological compartment will be implemented for the entire value-chain from acquisition platforms to data delivery. It will support the development of suitable technologies, offering dedicated access to an increased number of infrastructures, facilities (New TNA version), and services. In order to reach this target the JERICO-NEXT project is structured along 6 scientific areas addressing both scientific questions and policy/societal requirements.
The 6 key environmental Areas were prioritized because of their relevance to the descriptors of good environmental status of the MSFD, and to operational needs of coastal services. These Areas are :

1) pelagic biodiversity,

2) benthic biodiversity,

3) chemical contaminant occurrence and related biological responses,

4) hydrography and transport,

5) carbon fluxes and carbonate system,

6) operational oceanography.

The project fits a specific strategy, from which the abovementioned 6 scientific areas are derived. Indeed, in JERICO-NEXT observation methodologies and technologies will be developed or updated to specifically answer challenges related to specific environmental Areas, then tested in dedicated laboratory and/or field experiments in the framework of 6 Joint Research Activity Projects (JRAPs). The latter will provide a feedback and recommendations for further upgrades, and will serve as test case for the data flows, including towards the integration of several kinds of data flows (physics, biology and chemistry). Harmonization of observing systems, sensor calibration and maintenance procedures will also be focused according to these Areas.

JERICO-NEXT gathers a strong consortium of partners who are responsible for the majority of coastal observatories in Europe and have substantial experience in observations methods, R\&D of technologies and methodologies suitable to long term deployment with particular focus on the biology. We expect a significant enhancement of the capability and the quality of measurements in the coastal infrastructures.

In particular, innovative (semi)-automated observation techniques for addressing phytoplankton dynamics at high resolution and in (near) real-time will be investigated. Biosensors using Surface Plasmon Resonance to detect algae toxins and contaminants will be investigated as well. New platforms such as HF Radars and coastal profilers will be integrated. New HF-radar products will be delivered to the scientific community through innovative and cost-effective methods. The capabilities of coastal profilers will be enhanced through a series of developments and tests focusing in the biological component and the reliability of the systems.

Furthermore, the development of reliable high-precision and high-frequency sensors for the monitoring of key climate parameters (e.g. carbonate system in the coastal ocean) is of primary importance. Following up the work done in JERICO, some ongoing developments will be completed, providing at the same time operational platforms for testing technologies developed in other projects.

Building on FP7-JERICO achievements, efforts will be directed towards the development of an advanced OSE/OSSE infrastructure focusing on the high-resolution biochemical transport in coastal areas and using well-established standards for optimal configuration of OSSEs. 
JERICO-NEXT will establish links with national funding and regional agencies (e.g. JPI Ocean), which are financially supporting observing systems that are parts of the JERICO-RI, to ensure that they know the added value of their contribution. While agencies are typically very aware of the capital investments required for observing systems, in the medium to long term, operational and recurring costs can often be significant in the marine environment. It will make an assessment of both the capital and operational expenditure costs for the JERICO-NEXT infrastructure. In terms of a legal framework for the long-term implementation of JERICONEXT, an assessment will be conducted for the most commonly used legal forms (ERIC, AISBL) and a recommendation made as to which is the most suitable special purpose vehicle or legal entity for the RI. Finally, a comprehensive cost benefit and value analysis will be undertaken for JERICO-NEXT, outlining both the direct costs and benefits, but also the non-quantifiable costs and benefits associated with coastal marine observing systems. The measurable impact on interested communities of users will be an inherent part of this analysis. Successes recorded through the Virtual Access activity and Trans National Access will be used as a critical input factor in the cost benefit analysis. This will lead to the definition of a strategy for sustainability of JERICO-RI as a European Research Infrastructure. Different models and options for the sustainability of JERICO-RI through, for example alliances with other I3 and ESFRI infrastructures, and the integration into existing legal entities will be investigated.

\section{ACKNOWLEDGMENT}

The work was supported by the EU FP7INFRASTRUCTURES-2010-1, Towards a Joint European Research Infrastructure Network for Coastal Observatories JERICO, Grant Agreement Number 262584, coordinated by IFREMER. Special thanks are addressed to the JERICO European project officer, A. Robin, for her support and to the members of the JERICO Scientific Advisory Committee (SAC). The coordination team deeply thanks all the JERICO partners for a fruitful and successful collaboration.

\section{REFERENCES}

[1] European Marine Board, "Navigating the Future IV", Position Paper 20 of the European Marine Board, 2013, Ostend, Belgium. ISBN: 9789082093100

[2] G. Petihakis et al., "Harmonization in the Joint European Research Infrastructure Network for Coastal Observatories -JERICO 2015”, 2015, IEEE Journal of Oceanic Engineering, unpublished.

[3] W. Petersen, "FerryBox Systems : State-of-the-Art in Europe and Future Development", 2014, Journal of Marine Systems, 140, 4-12. DOI: 10.1016/j.jmarsys.2014.07.003.

[4] S. Pensieri, R. Bozzano, M.E. Schiano, M. Ntoumas., E. Potiris, C. Frangoulis, D. Podaras, G. Petihakis, "Promising tools to test and intercalibrate dissolved oxygen, fluorescence and turbidity sensors", 2015, Journal of Marine Systems (JERICO special issue), unpublished.

[5] M. Brumovský, J. Bečanová, J. Kohoutek, H. Thomas, W. Petersen, K. Sørensen, L. Nizzetto, "Exploring the occurerence and distribution of contaminantes of emerging concern through unmanned sampling from Ships of opportunity in th enorth sea", 2015, Journal of Marine Systems (JERICO special issue), unpublished.

[6] M. González Dávila, J. Magdalena Santana-Casiano, G. Petihakis, M. Ntoumas, M. Suarez de Tangil, E. Krasakopoulou, "Seasonal pH variability in the Saronikos Gulf: a year-study using a new photometric pH sensor", 2015, Journal of Marine Systems (JERICO special issue), unpublished. 